\title{
L’archéologie de la Grande Guerre : une nécessaire interdisciplinarité
}

World War I archeology: pluridisciplinarity at stake

\section{Michaël Landolt}

\section{(2) OpenEdition}

1 Journals

Édition électronique

URL : http://journals.openedition.org/artefact/817

DOI : $10.4000 /$ artefact. 817

ISSN : 2606-9245

Éditeur :

Association Artefact. Techniques histoire et sciences humaines, Presses universitaires du Midi

Édition imprimée

Pagination : 61-75

ISBN : 978-2-7535-7305-5

ISSN : 2273-0753

\section{Référence électronique}

Michaël Landolt, «L'archéologie de la Grande Guerre : une nécessaire interdisciplinarité », Artefact [En ligne], 6 | 2017, mis en ligne le 31 mai 2018, consulté le 10 décembre 2020. URL : http:// journals.openedition.org/artefact/817 ; DOI : https://doi.org/10.4000/artefact.817

\section{c)}

Artefact, Techniques, histoire et sciences humaines est mise à disposition selon les termes de la Licence Creative Commons Attribution - Pas d'Utilisation Commerciale - Pas de Modification 4.0 International. 


\section{L'archéologie de la Grande Guerre : une nécessaire interdisciplinarité}

Michaël LANDOLT*

\section{Résumé}

Les vestiges de la Grande Guerre sont désormais pris en considération par les archéologues. Bien qu'opportuniste à ses débuts, à la fin des années 1980, l'archéologie du premier conflit mondial a su développer des problématiques scientifiques à travers différentes approches. Au moment où s'éteignait le dernier vétéran du conflit, en 2011, la découverte du « Pompéi de la Grande Guerre » à Carspach (Haut-Rhin) a permis de mettre au jour un vaste abri souterrain où vingt et un soldats allemands avaient été ensevelis en mars 1918. Cette fouille a déclenché de nombreuses réflexions méthodologiques pluridisciplinaires autour de la discipline archéologique.

Mots-clés : archéologie, Grande Guerre, méthodologie, passé récent, pluridisciplinarité.

\section{Abstract. World War I archeology : pluridisciplinarity at stake}

A good number of French archaeologists who deal with the "recent past » are now working on vestiges from World War I. The opportunism of the earliest studies from the late 1980's notwithstanding, archaeologists engaged in this domain have developed differently approaches

*. Michaël Landolt est archéologue au Service régional de l'archéologie, à la DRAC Grand Est, après avoir travaillé au Pôle d'archéologie interdépartemental rhénan en Alsace. Ses thématiques de recherches concernent l'âge du Fer et l'époque contemporaine. Chercheur associé à l'UMR 7044 Archimède, il a contribué, depuis 2005, au développement de l'archéologie des conflits contemporains en participant à de nombreuses publications sur cette thématique. Ses recherches sur la Grande Guerre concernent principalement la vie quotidienne du combattant allemand à travers son alimentation. En 2011, il a dirigé la fouille du Kilianstollen, à Carspach (Haut-Rhin). Il a également participé à plusieurs expositions, comme $A$ l'Est, du nouveau! L'archéologie de la Grande Guerre en Alsace et en Lorraine, à Strasbourg, 14 Menschen Krieg, à Dresde, ou De terre et d'acier. Archéologie de la Grande Guerre, à Arras, Reims et Meaux. Ses thématiques de recherches sur la Seconde Guerre mondiale concernent la défense passive et les lieux d'internement nazis. Contact : [michael.landolt@culture.gouv.fr]. 
and expanded their research. In 2011, the year that saw the passing of the last WWI veteran, the discovery of « the Pompeii of World War I » in Carspach (Alsace) brought to light a large, underground shelter in which twenty-one Germans were buried in March 1918. The work done in Carspach represented a milestone for the discipline. It also led the archaeologist who headed that dig to reflect upon the methodology of his discipline.

Keywords : archeology, Great War, methodology, pluridisciplinarity, recent past.

Alors qu'ils ont été longtemps considérés comme des éléments perturbateurs ou une source de danger, les vestiges de la Grande Guerre sont aujourd'hui pris en considération par les archéologues. Bien qu'opportuniste à ses débuts, à la fin des années 1980, l'archéologie du premier conflit mondial a su développer des problématiques scientifiques, présentées à travers plusieurs publications synthétiques et expositions thématiques ${ }^{1}$. La recherche, d'abord centrée sur les pratiques funéraires, est aujourd'hui plus diversifiée et se confronte à l'ensemble des sources historiques disponibles, parfois en quantité considérable (témoignages, photographies, cartes, archives). La question de l'apport de l'archéologie à la connaissance de ce passé récent doit aujourd'hui être considérée comme réglée, de la même manière que plus personne ne nie aujourd'hui l'intérêt et la légitimité de l'archéologie médiévale et moderne ${ }^{2}$. En 2013, la ministre de la Culture et de la Communication décla- rait que les vestiges résultant des conflits armés du $\mathrm{xx}^{\mathrm{e}}$ siècle " doivent bénéficier $d^{\prime}$ une prise en compte et d'une protection identiques à celles des autres éléments du patrimoine archéologique ${ }^{3}$ ».

$\mathrm{Au}$ moment où s'éteignait le dernier vétéran du conflit, en 2011, la découverte du «Pompéi de la Grande Guerre ${ }^{4}$ » à Carspach (Haut-Rhin) a permis de mettre au jour un vaste abri souterrain où vingt et un soldats allemands avaient été ensevelis à la suite d'un bombardement, en mars 1918. En constituant la première fouille archéologique préventive française spécifiquement destinée à appréhender des vestiges de la Première Guerre mondiale, cette opération est devenue un jalon important de la discipline en France. Elle a également déclenché de nombreuses réflexions méthodologiques pluridisciplinaires, désormais abordées par de nombreux auteurs, autour de l'archéologie.

\section{La galerie allemande de Carspach : un référentiel}

L'abri de Carspach a été découvert lors de travaux d'aménagement routier. Des sources écrites et un monument commémoratif indiquaient la présence, sur l'emprise du projet, d'un abri construit à plusieurs mètres sous terre : une galerie allemande datant de la Première Guerre dénommée Kilianstollen (Galerie Kilian). 
En 2007, un diagnostic archéologique avait permis d'identifier les vestiges de tranchées allemandes de première ligne, ainsi qu'un escalier d'accès à la galerie. Plusieurs observations archéologiques ponctuelles réalisées lors des travaux de terrassement ont mené, en 2010, au dégagement partiel d'un tronçon de galerie dans un exceptionnel état de conservation. Cette découverte a entraîné la réalisation d'une fouille préventive en $2011^{5}$.

\section{Le Kilianstollen, un abri collectif de grande capacité}

Sur le flanc de la colline du Lerchenberg, le Kilianstollen se situe au niveau de la première ligne de front allemande qui s'est fixée à l'ouest d'Altkirch entre 1914 et 1918. Il s'agit d'un abri souterrain de grande capacité, construit entre 1915 et 1916, où les soldats qui occupaient les tranchées de première ligne pouvaient trouver refuge en cas de bombardement d'artillerie. La galerie orientée nord-sud, parallèle aux tranchées, mesure environ $125 \mathrm{~m}$ de long, $1,20 \mathrm{~m}$ de large pour $1,70 \mathrm{~m}$ de haut. Une centaine de mètres, localisée sur l'emprise de la déviation, a pu être intégralement fouillée. Le Kilianstollen a été construit à une profondeur située entre 3,50 et $6 \mathrm{~m}$ selon des techniques de travail minier. Le sédiment a été creusé aux dimensions des cadres jointifs constitués de madriers en bois assemblés par tenonmortaise. Neuf accès sur les seize attestés en 1918 ont pu être fouillés. Ils assuraient la liaison avec l'extérieur vers les tranchées et un chemin creux. Un système de plancher permettait l'écoulement des eaux d'infiltration. Il apparaît également que le Kilianstollen a fait l'objet de réaménagements jusqu'à la fin de la guerre. Par exemple, la mise en place de poteaux verticaux au centre de la galerie, qui semblent renforcer la stabilité du plafond sans cependant être nécessaires, est liée à l'installation de lits superposés condamnant la moitié du passage. Les tronçons de galerie ne comportant pas de lits présentaient des banquettes en bois où les soldats pouvaient patienter. L'existence de tablettes et de chaises a également été mise en évidence. Des portes étaient installées régulièrement à l'aplomb de chaque escalier ou pour compartimenter le long couloir de la galerie. Enfin, une pièce communiquant avec la galerie pourrait correspondre à une chambre destinée aux officiers. On note également la présence d'aménagements liés au rangement et au stockage (étagères, caisse, armoire). La galerie était chauffée par des poêles, alimentée en électricité et raccordée au téléphone.

\section{Les opérations du 18 mars 1918 et leurs conséquences}

L'historique du $94^{\mathrm{e}}$ régiment d'infanterie de réserve allemand (Reserve Infanterie Regiment 94) publié en 1934 et les archives françaises (Service historique de la Défense, Vincennes) renseignent sur les événements du 18 mars 1918 sur cette partie du front. Le matin, l'artillerie allemande bombarde les lignes françaises à l'aide d'obus à gaz, puis, l'aprèsmidi, l'artillerie française concentre ses tirs sur l'Ouvrage bulgare, dénomination française attribuée au secteur du front où se situait le Kilianstollen ${ }^{6}$. Ces deux opérations se déroulent le même jour par coïncidence car le bombardement français de la position était planifié depuis plusieurs 
mois. Le tir d'artillerie allemand est peut-être une manœuvre de diversion dans le cadre de l'offensive allemande en préparation dans la Somme. Suite à cette opération d'artillerie française, la plus grande partie de la $6^{\text {e }}$ compagnie du $94^{\text {e }}$ régiment d'infanterie de réserve trouve alors refuge dans la galerie considérée comme sûre. Vers 13h30, après avoir essuyé trois tirs successifs, la partie sud de la galerie, où la couche de terre est la plus mince, s'effondre sur environ $60 \mathrm{~m}$. On dénombre dix blessés et trentequatre soldats ensevelis. Entre $20 \mathrm{~h} 30$ et $21 \mathrm{~h} 30$, un groupe du $22^{\mathrm{e}}$ régiment $\mathrm{d}$ 'infanterie français s'approche avant de rejoindre ses positions.

Dès la tombée de la nuit, les soldats allemands tentent une opération de sauvetage afin de libérer d'éventuels survivants bloqués dans la galerie. Des corps et des blessés sont exhumés et vingt et un soldats restent portés disparus. Les recherches sont interrompues en raison de contraintes techniques (présence de terre dans la galerie effondrée, difficultés d'accès et de progression, poursuite des combats) (fig. 11, cahier couleur). Les soldats tués lors de cette journée, initialement inhumés à Wittersdorf (HautRhin), reposent aujourd'hui pour partie dans le cimetière militaire allemand d'Illfurth (Haut-Rhin) aménagé en 1920. Enfin, le départ du régiment, le 4 avril, pour les Flandres (Belgique) au Mont Kemmel entraîne l'arrêt définitif des recherches des corps.

\section{Le 18 mars 1918 à la lumière de faits semblables}

L'utilisation de galeries comme abris souterrains confronte le commandement allemand à de nombreux problèmes de sécurité7. Plusieurs expériences désastreuses sont à relever entre le printemps et l'été 1917. Au problème du regroupement de troupes dans un seul abri, pouvant entraîner d'importantes pertes en cas de destruction même partielle par des obus de gros calibre, s'ajoutaient des problèmes d'encombrement car l'étroitesse des sorties ne permettait pas la réoccupation rapide des positions lorsque l'ennemi tentait une approche terrestre. Le commandement allemand prescrit alors de ne plus réaliser de pareils aménagements où plusieurs centaines de soldats pouvaient trouver refuge lors d'une attaque. Ceux qui existaient auparavant ne sont cependant ni détruits ni abandonnés. Dans le sud de l'Alsace, secteur faiblement actif du front, ces aménagements souterrains continuent d'être utilisés, comme le démontrent la documentation d'archives et la fouille de Carspach. On citera quelques exemples emblématiques concernant des tunnels de bien plus grande capacité. Sur le front de Champagne, l'épisode tragique du Mont Cornillet, à proximité de Reims (Marne), mérite d'être évoqué. Ce vaste ouvrage souterrain allemand constitué de trois galeries parallèles a fait l'objet, le 20 mai 1917, de tirs d'artillerie français. $\mathrm{Au}$ total, plus de six cents soldats allemands y trouvèrent la mort. Plus tard, le bombardement français du 20 août 1917 sur le Kronprinz Tunnel long d'environ un kilomètre, sur les pentes du Mort-Homme près de Verdun (Meuse), 
entraîna le décès de plus de cent soixantedix soldats allemands.

\section{Le 18 mars 1918 à travers les observations de la fouille}

Les fouilles archéologiques ont révélé que le Kilianstollen a cédé au niveau de deux connexions entre la galerie et un escalier d'accès menant au chemin opposé à la ligne de front. En effet, le système d'assemblage des bois, plus complexe et fragile à ces emplacements, pourrait avoir cédé sous l'effet du bombardement français. Les vestiges de l'opération de secours ont été mis en évidence. Certains tronçons non effondrés ont fait l'objet d'un étaiement permettant la consolidation de la galerie. La fouille a pu démontrer que la progression des opérations de secours a été rendue impossible par l'effondrement d'un côté de la galerie qui avait intégralement cédé sous le poids du sédiment. En effet, un enchevêtrement de bois et d'objets divers obligeait à dégager un accès depuis la surface, qui n'a pu être mis en œuvre pendant les combats. Les vingt et un soldats disparus du 18 mars 1918, piégés sous l'écrasement de la galerie, sont probablement décédés très rapidement à cause de l'effet de blast, une surpression liée aux explosions, et de l'effondrement partiel de la galerie. En plus des équipements militaires réglementaires, de nombreux objets personnels ont été retrouvés sur ou à proximité des défunts. Ils se rapportent principalement à la toilette, l'hygiène, la religion, les superstitions, le jeu, le tabac, l'argent, la couture, la nourriture et l'écriture. On notera la très bonne conservation des matériaux organiques, notamment le bois et le cuir, et celle partielle des tissus et papiers. Plusieurs livrets militaires et plaques d'identité retrouvés en association avec les corps ont permis l'identification des disparus.

\section{Les traces d'une réutilisation de la galerie}

Les fouilles démontrent que la partie non endommagée de la galerie a fait l'objet de réaménagements après l'effondrement du 18 mars 1918, qui permettent d'envisager son occupation jusqu'à la fin de la guerre. Certains tronçons ont ainsi été réutilisés en abris indépendants ne communiquant plus entre eux. Un escalier supplémentaire a même été implanté et les communications avec les parties effondrées ont été obstruées. Cette proximité avec des corps de soldats enfouis trouve quelques parallèles, dans des proportions toutefois différentes, au Fort de Douaumont (Meuse) par exemple, où, suite à une violente explosion qui se produisit le 8 mai 1916, environ sept cents soldats allemands périrent. Une grande partie d'entre eux a alors été emmurée dans un tronçon encore en fonction du fort car il était impossible de les inhumer à l'extérieur. D'autres exemples sont connus, ainsi dans l'iconographie, pour des sapes ou des galeries.

Suite à ces réaménagements, un système de pompe électrique a probablement été mis en place afin d'évacuer les eaux d'infiltration qui ne pouvaient plus être régulées par la légère pente de la galerie désormais partiellement obstruée. Enfin, les traces d'un incendie ont également pu être observées alors que la galerie était en grande partie envahie par l'eau après l'arrêt du pompage. Cette destruction a probablement été causée 
par des bouteilles incendiaires, comme en témoigne la bouteille en verre remplie de liquide inflammable retrouvée au pied d'un escalier partiellement calciné. Cette destruction pourrait avoir été menée par l'armée allemande lors de l'évacuation de l'Alsace, pendant les quinze jours qui suivirent l'Armistice, ou lors de la remise en état des terrains par les agriculteurs juste après le conflit.

\section{Un seul événement, trois monuments successifs}

Afin de rendre hommage aux vingt et un soldats piégés dans la galerie, trois monuments successifs ont été érigés. Le premier en bois, construit dès mars 1918 à proximité d'une des entrées de la galerie, n'est connu que par une cartephoto d'époque et une représentation dans l'historique du $94^{\mathrm{e}}$ régiment d'infanterie de réserve. Il comportait la liste des soldats disparus avec leurs grades, dates et lieux de naissance.
Afin de remplacer ce monument provisoire, un bloc en calcaire sculpté a été mis en place. Ce dernier, dont l'existence avait été oubliée, a été redécouvert lors des fouilles. On notera qu'il a fait l'objet d'une destruction volontaire. L'analyse de son iconographie montre de grandes parentés avec l'insigne des blessés allemands (Verwundetenabzeichen), créé le 3 mars 1918. La datation de ce monument pose problème. Il pourrait dater de la Grande Guerre ou avoir été réalisé vers 1941-1943, pendant l'annexion de l'Alsace à l'Allemagne.

Enfin, le troisième monument, également en pierre, portant les noms des vingt et un disparus a été inauguré le 27 mai 1962. Ce dernier monument, initialement situé sur le tracé de la route, se trouve aujourd'hui dans le cimetière militaire allemand d'Illfurth. Après l'étude anthropologique, les corps des vingt et un individus ont été inhumés en juillet 2013 dans ce cimetière.

\section{Les problématiques liées aux spécificités du contexte et les réflexions méthodologiques}

\section{Les spécificités du contexte de Carspach}

La fouille de Carspach présente de nombreuses spécificités. Suite à une catastrophe, une grande partie du site a été figée à un instant $\mathrm{T}$ de l'après-midi du 18 mars 1918 et des quelques jours qui suivirent. Les observations de fouille peuvent en grande partie être mises en relation avec les témoignages écrits rela- tant les événements du bombardement français puis l'effondrement de la galerie allemande jusqu'à la mise en œuvre des secours. Il est très rare de pouvoir rattacher un contexte archéologique à un événement d'aussi courte durée décrit par $d$ 'autres sources. Les recherches menées dans différents fonds d'archives ont en effet permis de retrouver des documents concernant la construction, l'utilisation et la destruction de la galerie, qui vien- 
nent compléter les observations de terrain (plans, inventaires, plan de travail) (fig. 12, cahier couleur). Les documents liés à la construction de la galerie peuvent être mis en relation avec les vestiges. Ils informent sur le nombre de pionniers et de soldats nécessaire au chantier, les jours travaillés, les conditions météorologiques, ainsi que les problèmes techniques qui ont pu être rencontrés. La construction de la galerie depuis chaque extrémité, à travers deux chantiers, est confirmée par la mise en évidence d'un léger décalage d'orientation et de niveau pour la partie centrale de la galerie. La date de construction de la galerie pendant l'hiver 1915-1916 est quant à elle bien confirmée par l'étude dendrochronologique ${ }^{8}$. Les témoignages retranscrits dans les archives allemandes et françaises doivent faire l'objet d'un examen critique. Par exemple, il faut corriger le plan de 1916 comportant quelques erreurs et relativiser la capacité théorique de cinq cents soldats attestée dans un document d'archives daté de janvier 1916, car il est, en effet, difficile $\mathrm{d}$ 'installer plusieurs centaines de soldats intégralement équipés dans $150 \mathrm{~m}^{2}$ déjà partiellement occupés par de nombreux aménagements.

\section{La valeur du témoignage}

Dans le cadre de l'archéologie contemporaine française, la question de la valeur du témoignage s'inscrit dans la lignée des préoccupations des archéologues américains confrontés depuis une trentaine d'années au problème de la concordance/divergence des témoignages avec les vestiges retrouvés lors des fouilles de sites du passé récent ${ }^{9}$. Les observations de fouille peuvent en grande partie être mises en relation et confrontées avec les témoignages retranscrits relatant les événements, de l'effondrement de la galerie à la mise en œuvre des secours.

Si la présence des vingt et un soldats ensevelis est une réalité qui a pu être confirmée, l'effondrement de la galerie a pu être mieux appréhendé par la fouille. Les sources écrites indiquaient que la partie de la galerie où la couche de terre est la plus mince, s'était effondrée sur environ $60 \mathrm{~m}^{10}$. Cependant, la fouille a démontré que la galerie s'était effondrée au niveau de deux tronçons indépendants d'une vingtaine de mètres chacun. L'ancrage profond du souvenir de ce tragique événement, devenu légendaire dans la mémoire collective locale, reste à étudier et à mettre en regard avec les observations issues des différentes sources.

Les limites du témoignage ont fait l'objet de nombreuses études, notamment au début du $\mathrm{xx}^{\mathrm{e}}$ siècle. Initialement menées par des médecins, psychologues et juristes dans un cadre judiciaire, elles ont ensuite été reprises par les historiens comme Marc Bloch (1886-1944) qui fut confronté pendant la Grande Guerre aux rumeurs issues de l'imagination collective $^{11}$. La tentative de crime imaginée par le criminologiste autrichien Franz von Liszt (1851-1919), simulée en 1901, a démontré que la valeur du témoignage dépendait de la nature des faits, des aptitudes ou des réactions du témoin et du temps qui existait entre le fait et sa description ${ }^{12}$.

\section{Archéologie de la violence}


Par ailleurs, les corps retrouvés dans la galerie de Carspach ne sont pas à mettre en relation avec une pratique funéraire. Aucune manipulation n'a été effectuée après la mort et les positions des soldats ont été figées au moment de l'effondrement de la galerie (couché, assis, debout, recroquevillé) (fig. 13, cahier couleur). Ce type de contexte archéologique, caractérisé par des hommes saisis par une mort violente provoquée par une catastrophe humaine ou naturelle, où il n'y a pas de ré-intervention humaine, est extrêmement rare : des cas similaires sont connus, par exemple, pour la Protohistoire (Vallée de Tollense) ou l'Antiquité (Pompéi, Herculanum, Kourion, Midéa). L'absence de ré-intervention post-mortem dans les secteurs effondrés de la galerie permet de connaître les aménagements internes de l'abri collectif et l'équipement des soldats.

\section{L'équipement du soldat}

Au-delà de l'aspect émotionnel, le mobilier découvert en association avec les corps des soldats présente un intérêt historique $^{13}$. Ce dernier, d'abord utile aux identifications, fournit des indications relevant du domaine de la guerre économique en permettant de mettre en évidence l'importance du phénomène des succédanés, par exemple, dans les armements et équipements individuels. L'archéologie apporte une contribution à la connaissance de l'état réel des uniformes. En effet, des découvertes donnent un aperçu de ce que portaient réellement les combattants et constituent une source d'informations, complémentaire aux autres (notices descriptives, clichés officiels, illustrations parues dans la presse, souvenirs photographiques, etc.). Ainsi, le corpus du Kilianstollen permet de connaître le niveau d'équipement de vingt et un militaires allemands appartenant à une même compagnie. Un fragment de veste de type Feldrock $M^{\text {le }} 1907 / 10$ (ou d'une de ses variantes) atteste du port de cet effet encore en 1918. Il confirme que la vareuse (Bluse), officialisée par un ordre du cabinet impérial du 21 septembre 1915, si elle a connu une généralisation à partir de la fin de 1916 et au début de 1917, n'a pas exclu les panachages d'effets de différents modèles, observés jusqu'à la fin du conflit. De manière similaire, des trouvailles permettent de tirer des enseignements sur l'existence ou, au contraire, l'absence d'articles ou de matériels dans l'environnement du soldat à une période déterminée.

\section{Les dépotoirs : un potentiel encore sous-estimé}

L'étude des dépotoirs situés à proximité immédiate de la galerie apporte des informations inédites pouvant alimenter de nouvelles problématiques historiques. Le mobilier retrouvé permet d'appréhender différentes activités : l'alimentation, l'écriture, la santé, l'hygiène, le jeu ou l'artisanat. Les problématiques alimentaires se développent peu à peu en France depuis quelques années ${ }^{14}$. Le caractère anecdotique ou général de certains phénomènes observés à travers l'archéologie doit être pris en compte car il existe plusieurs réseaux d'approvisionnement en nourriture : intendance militaire, boutiques militaires et civiles, colis civils, récupération dans les maisons abandonnées ou évacuées, ou auprès 
de l'ennemi et réquisition ou même production par les combattants eux-mêmes (tableau 1). Comme pour les autres périodes, les corpus étudiés par les archéologues sont incomplets à cause du recyclage de la matière première, notamment du métal et du verre, de la réutilisation et du détournement (contenants consignés, détournement de la fonction de l'objet à d'autres fins, matière première pour l'artisanat), ou de l'incinération d'une part importante des déchets. Le mobilier enfoui dans les dépotoirs militaires retrouvé constitue donc une représentation partielle et sélective de la consommation des soldats. Le développement de la recherche archéologique sur l'alimentation du soldat de la Grande Guerre devra mettre en avant des contextes fouillés scientifiquement et bien datés afin d'avancer des hypothèses à mettre en relation avec la documentation issue des archives. Les études sur ce type de mobilier sont encore aujourd'hui bien souvent anecdotiques et factuelles, aucune approche quantitative et évo- lutive ne pouvant encore être proposée dans l'état actuel de la documentation.

La consommation des boissons peut être étudiée à travers l'étude des bouteilles même si le contenu est parfois différent de ce qu'induisent la forme ou l'inscription portée par le récipient. Les sources d'approvisionnement s'expliquent généralement par la proximité des lieux de production. Ainsi, en Alsace, les brasseries strasbourgeoises sont très bien représentées dans les secteurs allemands (Fischer, Freysz, Gruber, Münsterbräu, Prieur, Schutzenberger). De nombreux spiritueux régionaux sont observés dans les dépotoirs allemands : Heidelbeerwein de Nuremberg (Bavière), alcool de gentiane de Bavière, alcool de genévrier de la marque Steinhäger provenant de la région de Steinhagen (Rhénanie-du-Nord-Westphalie), alcool de cumin (Kümmel) de la firme Gilka de Berlin, Goldwasser de Dantzig (Prusse occidentale), alcool d'arak à base de jus de raisin distillé aromatisé aux graines d'anis (Küstennebel) du Nord

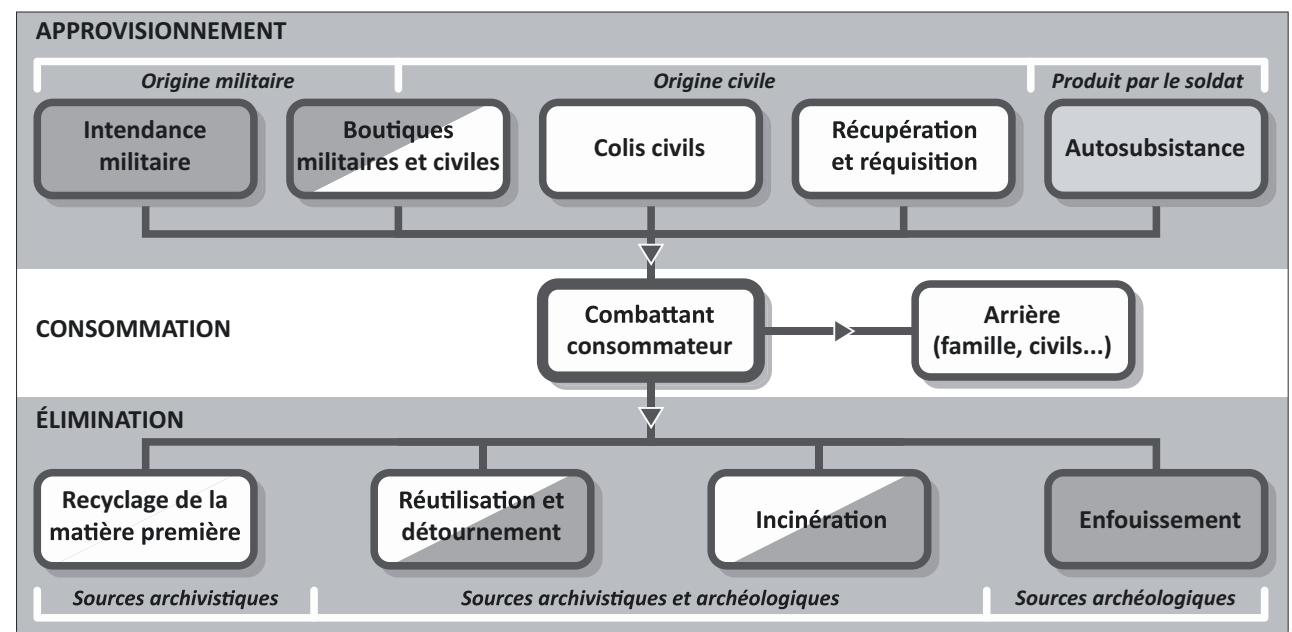

Tableau 1. - Schéma de l'approvisionnement du combattant pendant la Grande Guerre. Document M. Landolt, MCC. 
de l'Allemagne (Schleswig-Holstein). Cette diversité témoigne parfois de la provenance géographique des soldats. L'étude du lien éventuel entre l'origine des troupes et des approvisionnements reste encore cependant à entreprendre et développer.

La mise en évidence de certains aliments originaux lors des fouilles peut parfois être mise en relation avec des fêtes ou événements particuliers. Par exemple, la découverte d'huîtres et d'escargots à Carspach peut être mise en relation avec un ou plusieurs repas liés à un événement festif (comme Noël (15) $^{15}$ (fig. 14, cahier couleur). La consommation de viande de chien, même si elle est marginale sur le même site, peut être liée à la cynophagie attestée en Allemagne, par exemple en Bavière et en Saxe au début $\mathrm{du} \mathrm{xx}^{\mathrm{e}}$ siècle $^{16}$. Ainsi, cette pratique culinaire serait imputable aux soldats saxons qui occupaient la position en mars $1918^{17}$.

Le cas des bouteilles à bille est également emblématique car il nous interroge sur la durée d'utilisation de certains types d'objets et sur leur oubli en quelques générations seulement. Ce type de contenant en verre, au profil singulier, où une bille en verre joue le rôle de bouchon grâce au gaz carbonique contenu sous pression dans la boisson (limonade ou eau minérale), apparu au Royaume-Uni vers 1872 , est bien attesté chez plusieurs belligérants pendant la Grande Guerre avant de disparaître à la veille du second conflit mondial ${ }^{18}$ (fig. 15, cahier couleur). Sa courte durée d'utilisation, à l'échelle de deux générations, interpelle car, aujourd'hui, quelques générations plus tard, cet objet particulier n'appartient plus aux référentiels de la société européenne actuelle même si ce dernier existe encore aujourd'hui en Asie, au Japon (Ramune) et en Inde (Banta). Certaines variantes, retrouvées sur le front ouest dans des positions allemandes, présentent des inscriptions en anglais, espagnol ou portugais faisant référence à des régions $\mathrm{d}$ 'Afrique et d'Amérique latine (Afrique du Sud, Argentine, Brésil, Chili, Paraguay, Pérou, Venezuela ${ }^{19}$ ). Leur présence sur le front est liée au blocus mis en œuvre pendant le conflit par les Alliés. Devant la pénurie croissante de matière première, les industriels allemands s'étaient résignés à utiliser sur le front occidental des contenants élaborés en Allemagne mais initialement destinés à l'exportation, malgré leurs libellés en langues étrangères. Cet exemple illustre les schémas interprétatifs simplistes qui auraient pu être proposés en l'absence d'autres sources d'archives : par exemple, des bouteilles originaires d'Amérique latine ou d'Afrique méridionale exportées avec leur contenu en Europe retrouvées sur leur lieu de consommation en Europe.

Les études archéologiques tendent à mettre en évidence une dichotomie entre les quantités importantes de certains aliments identifiés dans les dépotoirs par rapport à leur absence ou rareté dans d'autres sources textuelles ou iconographiques. Ainsi, les fouilles archéologiques mettent en évidence, côté allemand, une importante consommation de moutarde qui n'apparait pas dans les autres sources. Malgré l'abondante documentation photographique liée au premier conflit mondial, les documents où figurent des contenants liés à ce type de condiment sont en effet très rares $^{20}$. 


\section{Les interprétations}

Les problèmes d'interprétation des découvertes se posent également pour certains contextes. Le dépôt d'une chèvre non consommée, retrouvée en connexion dans un dépotoir de Carspach, illustre bien la problématique bien connue des dépôts d'animaux que l'on connaît, par exemple, pour l'âge du $\mathrm{Fer}^{21}$. Ce type de pratique est bien souvent trop facilement qualifié de cultuel en l'absence de réflexion plus poussée à partir de différentes hypothèses. À Carspach, l'hypothèse d'un animal lié à la production laitière qui n'aurait pas été consommé pour des raisons d'hygiène (animal impropre à la consommation tué lors d'un bombardement chimique, par exemple) ou parce qu'il aurait fait l'objet d'une relation particulière avec les hommes (animal de compagnie), peut être proposée, sans qu'il soit possible de l'affirmer avec certitude ${ }^{22}$.

Dans le cadre de l'étude des uniformes et de l'équipement du combattant, l'étude du mobilier retrouvé en association avec les soldats décédés dans la galerie de Carspach permet de mettre en évidence des adaptations parfois non réglementaires, des pratiques individuelles ou collectives généralement peu mises en valeur dans les collections et les musées militaires. À Carspach, la présence dans les porte-monnaie ou en association avec des chapelets de balles françaises, mise en évidence à plusieurs reprises (pour au moins cinq individus sur vingt et un), témoignerait de la généralisation de superstitions destinées à conjurer le mauvais sort ${ }^{23}$ (fig. 16, cahier couleur).

\section{Les chaînes opératoires}

Pendant les périodes d'inaction, les soldats se tournent vers le bricolage et trouvent sur le champ de bataille des matières premières qu'ils peuvent travailler sur le front, dans les cantonnements et à l'arrière, avec un outillage plus ou mois élaboré (douilles, éclats, boîtes de conserves, ossements, etc.). Certaines productions, parfois mercantiles, se structurent autour de véritables petits ateliers artisanaux, parfois reconnus lors des fouilles archéologiques. Parallèlement, certains objets sont produits en séries par des civils au sein de manufactures. Les méthodologies d'études des objets finis, des chutes et des rebus mises en place par les archéologues permettent de mieux identifier les contextes de réalisation de ces objets bien connus des collectionneurs, mais dont la chaîne opératoire reste encore imprécise à cause de la rareté des sources archivistiques ${ }^{24}$.

\section{Des référentiels pluridisciplinaires}

Les études pluridisciplinaires, qui se développent dans le domaine de l'archéologie de la Grande Guerre, permettent aussi de mettre en place des référentiels méthodologiques et expérimentaux utiles à de nombreuses disciplines (anthropologie, parasitologie, cémentochronologie, entomologie, étude des isotopes, quantification du mobilier, géomorphologie, etc. ${ }^{25}$ ). Le contexte de Carspach, quasiment unique à l'heure actuelle, présente ainsi, par exemple, un très grand intérêt méthodologique pour l'anthropologie en permettant d'enri- 
chir les bases théoriques de la discipline. Certaines méthodes utilisées de nos jours dans le cadre des analyses biologiques et taphonomiques peuvent en effet être vérifiées. À Carspach, l'étude anthropologique a été réalisée en aveugle, c'està-dire sans connaissance des données extérieures, afin de ne pas nuire à l'objectivité du travail (élément d'uniforme, lisibilité des plaques d'identité). Par ailleurs, l'étude de cémentochronologie, basée sur l'observation des phénomènes cycliques du tissu dentaire ou cément, a montré une forte corrélation entre l'âge réel et l'âge biologique estimé26.

Par ailleurs, les nombreuses observations taphonomiques réalisées sur la dégradation des structures archéologiques et sur des types de matériaux diversifiés (bois, cuirs, textiles, matériaux composites), parfois non attestés dans des contextes archéologiques plus anciens, peuvent être utiles à la compréhension des processus de dégradation et permettent de mettre en place de nouveaux protocoles de conservation et de restauration ${ }^{27}$. L'étude du remplissage des structures de la Grande Guerre, appréhendée à travers la géomorphologie, permet la mise en place de référentiels qui pourront être utiles à l'étude de périodes plus anciennes ${ }^{28}$. Enfin, l'étude des paysages fortement marqués par la Grande Guerre tend à se développer depuis quelques années, notamment grâce à l'apport des nouvelles technologies comme le Lidar ${ }^{29}$.

Les exemples issus de contextes contemporains viennent enrichir la réflexion sur la discipline archéologique et sur la valeur qu'il convient d'accorder aux observations, interprétations et hypothèses qu'elle génère, à travers la reconnaissance des limites liées aux conditions d'enfouissement, contextes et objets d'étude. Ils nous alertent sur certaines interprétations trop hâtives ou stéréotypées, parfois proposées par les archéologues, pouvant être facilement réfutées par l'étude d'autres sources. Il s'avère d'ores et déjà que les sources archivistiques ont permis de modérer à Carspach certaines interprétations archéologiques ou d'infirmer certaines hypothèses. Ces éléments permettent de nous questionner sur les nombreuses limites des reconstitutions théoriques des sociétés anciennes sans écriture, dont l'appréhension est uniquement issue des vestiges et des artefacts. De même, certains événements ponctuels, voire anecdotiques, laissent d'importantes traces matérielles qui peuvent très facilement faire l'objet d'interprétations généralistes. L'exploitation des données issues des fouilles archéologiques, qui tendent aujourd'hui à se développer, apportent également de nombreux éléments à des thématiques parfois peu étudiées à travers les autres sources de l'histoire. Il conviendra de développer ces travaux à travers des études pluridisciplinaires dont cet article vise à démontrer l'intérêt. 


\section{Notes}

1. Yves Desfossés, Alain Jacques, Gilles Prilaux, L'archéologie de la Grande Guerre, Rennes, Éditions Ouest-France-INRAP, 2008; Gilles Bellan, Florence Journot, Archéologie de la France moderne et contemporaine, Paris, La découverte, 2011 ; Stéphanie JACQUemot, Jean-Pierre LegENDRE (dir.), Vestiges de guerres en Lorraine. Le patrimoine archéologique des conflits mondiaux, Metz, Éditions Serpenoise, 2011; Bernadette SchnitzLeR, Michaël LANDolt (dir.), avec la coll. de Stéphanie JACquemot, Jean-Pierre Legendre, Jean-Claude Laparra, À l'Est, du nouveau! Archéologie de la Grande Guerre en Alsace et en Lorraine, catalogue d'exposition, Strasbourg, musées de la ville de Strasbourg, 2013; Michaël Landolt, « Des vingt et un de Saint-Rémy-la-Calonne (Meuse) aux vingt et un de Carspach (Haut-Rhin) : 1991-2011. Quelles problématiques pour l'archéologie de la Première Guerre mondiale en Alsace? ", Revue d'Alsace, 139, 2013, p. 17-343; Thomas ВRоск, Archäologie des Krieges. Die Schlachtfelder der deuschen Geschichte, Darmstadt, Philipp von Zabern, 2015.

2. Jean Chapelot, François Gentilli, «Trente ans d'archéologie médiévale en France », in Jean Снареlot (dir.), Trente ans d'archéologie médiévale en France. Un bilan pour un avenir, Caen, Publications du CRAHM, 2010, p. 3-24.

3. Réponse à la question écrite $\mathrm{n}^{\circ} 2768$ de M. Jean-Louis Christ, député du Haut-Rhin, à $\mathrm{M}^{\text {me }}$ Aurélie Filippetti, ministre de la Culture et de la Communication, Journal officiel, 15 janvier 2013, p. 427.

4. Cette terminologie a été employée à raison par la presse lors de la fouille. Les sites de Pompéi et de Carspach présentent en effet des similitudes de contextes car ils ont été ensevelis à la suite d'un événement particulier : éruption volcanique et bombardement. Les deux sites ont ainsi été figés dans un temps rapide.

5. Michaël LANDolt, "Archéologie de la Grande Guerre. La galerie allemande du Kilianstollen à Carspach ", Archéologia, mai 2012, $n^{\circ} 499$, p. 30-44; id., « Der Kilianstollen, eine deutsche Stollenanlage aus dem Ersten Weltkrieg bei Carspach (Elsass, Frankreich) ", in Archäologie im 19. und 20. Jahrhundert, Mitteilungen der DGAMN, 28, 2015, p. 135-146. www.14-18kilianstollen.eu.

6. Julius Richter, Das Reserve Infanterie Regiment 94 im Weltkriege 1914-1918. Nach den Kriegsakten des Regiments und nach persönlichen Aufzeichnungen, Jena, Neuenbahn, 1934, p. 287289. Service historique de la Défense : 22N2357.
7. Michaël LANDolt, Alexandre Bolly, avec la coll. d'Emmanuel DubaIL, Jean-Claude LAPARRA, " Deutsche Stollenanlagen im Oberelsass ", in Gerhard BAUER, Gorch PIEKen, Mathias Rogg (dir.), 14 Menschen Krieg, catalogue d'exposition, Sandstein, Dresden, Militärhistorisches Museum der Bundeswehr, 2014, p. 244-253.

8. Willy Teger, Dietrich HaKelberg, « La dendrochonologie ", in B. SchNitzLer, M. LANDOlt (dir.), À l'Est, du nouveau !, op. cit., p. 111-113.

9. Douglas D. Scott, "Oral Tradition and Archaeology : Conflict and Concordance Examples from Two Indian War Sites », Historical Archaeology, 2003, 37(3), p. 55-65 ; Michaël LANDOLT, "Quelle valeur accorder au témoignage? », in B. SchNitzLeR, M. LANDolt (dir.), À l'Est, du nouveau! , op. cit., p. 76-77.

10 J. Richter, Das Reserve Infanterie Regiment 94 im Weltkriege 1914-1918..., op. cit., p. 288.

11. Marc BLOCH, «Réflexions d'un historien sur les fausses nouvelles de la guerre ", Revue de synthèse historique, 33, 1921, p. 13-35.

12. Sally JAFFA, " Ein psychologischer Experiment im kriminalistischen Seminar der Universität Berlin ", Beiträge zur Psychologie der Aussage, I, 1903, p. 79-99. Lors de l'expérience de Franz von Liszt, les spectateurs assistèrent à la scène sans se douter qu'il s'agissait d'un événement joué destiné à étudier la psychologie du témoignage. La confrontation de la réalité avec la description des témoins montra que de nombreux détails descriptifs sur l'enchaînement des événements, les lieux ou les personnages étaient erronés ou divergents. D'autres expériences ont été menées à la même époque par le psychologue allemand William Sterne (1871-1938), les médecins belges Louis Vervaeck (1872-1943) et Auguste Ley (1873-1956), le médecin suisse Édouard Claparède (1873-1940) ou le juriste français François Gorphe (1889-1959).

13. Jean-Claude Laparra, Michaël Landolt, « Uniformes, équipements et armes : l'apport des découvertes archéologiques ", in B. SCHNitzLeR, M. LANDolt (dir.), À l'Est, du nouveau!, op. cit., p. 91-92.

14. Michaël LANdolt, Frank LesjeAn, « L'alimentation du soldat allemand sur les fronts d'Alsace et de Champagne à travers l'approche archéologique des dépotoirs de la Première Guerre mondiale ", Cahiers alsaciens d'archéologie d'art et d'histoire, LII, 2009, p. 139-159; id., " Der Alltag deutscher Soldaten im Ersten Weltkrieg ", in G. BAuer, G. PieKen, M. RogG (dir.), 14 Menschen Krieg, op. cit., p. 278-285; Michaël Landolt, Frank LesJeAn avec la coll. d'Alexandre BolLy, Matthieu 
Le Bailly, Olivier Putelat, « L'alimentation des combattants de la Grande Guerre à travers 1'archéologie ", in Caroline PoulaIN (dir.), Manger et boire entre 1914 et 1918, Dijon/Gand, bibliothèque municipale de Dijon/Snoeck, 2015, p. 86-95.

15. Jean-Claude Fombaron, Michaël LANDolt, "Fêter et commémorer ", in B. SchNitzler, M. LANDolt (dir.), À l'Est, du nouveau!, op. cit., p. 232-233; Olivier Putelat, Alexandre Bolly, Michaël LANDOLT, " La relation homme-animal sur le front alsacien pendant la Grande Guerre à travers l'archéozoologie ", Ethnozootechnie, 2015, 95, p. 49-63.

16. Marcel Hébert, « Le chien en Préhistoire. Le chien dans la mythologie », Bulletin de la Société préhistorique de France, 7-11, 1910, p. 556-558; Jacqueline Milliet, « Manger du chien? C'est bon pour les sauvages! ", L'Homme, 35-136, 1995, p. 81-82; O. Putelat, A. Bolly, M. Landolt, « La relation homme-animal... », op. cit.

17. Michaël LAndolt, Jean-Claude LAparra, Amélie PÉlissier, " Des études pluridisciplinaires au service de l'identification des soldats ", in B. Schnitzler, M. LANdolt (dir.), À l'Est, du nouveau!, op. cit., p. 108-110.

18. Michaël LANDolt avec la coll. d'Alexandre Bolly, Frank LesjeAN, Denis Mellinger, « Le contenant alimentaire en verre pendant la Première Guerre mondiale : une spécificité de l'approvisionnement des troupes allemandes ", in Véronique Arveiller, Hubert CABART (dir.), Le verre en Lorraine et dans les régions transfrontalières, actes du colloque de l'Association française pour l'archéologie du verre, 18-19 novembre 2011, Metz, Montagnac, Éditions Monique Mergoil, 2012, p. 5-7 (Monographies Instrumentum, ${ }^{\circ} 42$ ).

19. Alain JAcques, Michaël LANDOLT, «Une véritable pénurie pour les contenants en verre ", in B. Schnitzler, M. LANdolt (dir.), À l'Est, du nouveau!, op. cit., p. 188-189.

20 M. LANDOlt avec la coll. d'A. Bolly, F. LesJeAn, D. MELlinger, « Le contenant alimentaire en verre pendant la Première Guerre mondiale... », op. cit., p. 10-12.

21. Olivier Putelat, Michaël Landolt, « La caractérisation des dépôts animaux de La Tène ancienne à Geispolsheim "Schwobenfeld" (BasRhin) ", in Ginette Auxiette, Patrice Méniel (éd.), Les dépôts d'ossements animaux en France, de la fouille à l'interprétation, actes de la table-ronde de Glux-en-Glenne, 15-17 octobre 2012, Montagnac, Éditions Monique Mergoil, 2013, p. 25-68.

22. Olivier Putelat, "Une chèvre du Sundgau à Carspach? », in B. Schnitzler, M. LANdolt (dir.), À l'Est, du nouveau!, op. cit., p. 254-255.
23. Jean-Claude Fombaron, Michaël LAndolt, «Les pratiques religieuses et les superstitions ", in B. Schnitzler, M. Landolt (dir.), À l'Est, du nouveau!, p. 245-247.

24. Fabien GRIESSEL, «Soldatenkunst - auch ein deutsches Phänomen. Von Krieg, Granatenhülsen und Fingerringen ", in Archäologie im 19. und 20. Jahrhundert, Mitteilungen der DGAMN, $\mathrm{n}^{\circ} 28,2015$, p. 147-154.

25. Frédéric AdAM, Amélie PÉLIssier, «L'anthropologie », in B. SCHNITZLER, M. LANDOLT (dir.), À l'Est, du nouveau!, op. cit., p. 100-102; Émile Decker, Michaël LANDOLT, « L'étude des récipients en céramique et en verre : des méthodologies nouvelles à intégrer à la recherche ", in B. SCHNITZLER, M. LANDOLT (dir.), op. cit., p. 87-90; Jean-Bernard HuchET, « L'archéo-entomologie : les insectes nécrophages associés aux soldats de Carspach ", in B. Schnitzler, M. LANdolt (dir.), op. cit., p. 109-110; M. LANDOLT avec la coll. d'A. Bolly, F. Lesjean, D. Mellinger, « Le contenant alimentaire en verre pendant la Première Guerre mondiale... », op. cit., p. 15; Matthieu LE BAILly, " La parasitologie ", in B. SCHNITZleR, M. LANDOLT (dir.), op. cit., p. 104-105.

26. Amélie Pélissier, Benoît Bertrand, Matthieu LE BAIlly, Michaël LANDolt, « La fouille du Kilianstollen à Carspach (Haut-Rhin) : réflexions méthodologiques pluridisciplinaires au service de l'identification de soldats de la Première Guerre mondiale », actes des $8^{\text {es }}$ rencontres du Gaaf, « La mort de plus en plus proche. Rencontre autour de nos aïeux », 25 au 27 mai 2016, Marseille, à paraître.

27. Gaëlle Harouard, Solenne Milbled, Agathe Mulot, « Le mobilier des conflits contemporains : comment conserver des objets et matériaux com-

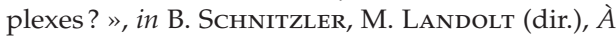
l'Est, du nouveau!, op. cit., p. 119-124; Stéphanie JACQUEMот, " La taphonomie des structures : l'apport de l'archéologie à l'étude du passé récent ", in B. Schnitzler, M. LANDolt (dir.), op. cit., p. 116; Marie-Jeanne ScHOLL, « La corrosion des plaques d'identification militaires en zinc de Carspach : une étude en cours », in B. Schnitzler, M. LANdolt (dir.), op. cit., p. 126127; Marie-Jeanne SCHOLL, «Caractérisation des plaques d'identification militaires en zinc provenant du site de Carspach (Alsace, Haut-Rhin, F) », CeROArt [En ligne], 2014, mis en ligne le 21 mars 2014, consulté le 9 octobre 2015. URL : [http:// ceroart.revues.org/4059].

28. Alain Devos, Pierre Taborelli, Mylène Dodici, Laurent Chalumeau, Jérôme Buridant, Nicolas Bollot, Anne Combaud, Yves Desfossés, « Rôle des conditions géographiques sur l'organi- 
sation spatiale des réseaux de défense de la Grande Guerre. Application à la Champagne (région de Reims) », Physio-Géo, 2015, 9-1, p. 157-174.

29. Pierre Taborelli, Alain Devos, Sébastien Laratte, Jérôme Brenot, Nicolas Bollot, Benjamin CancÈs, Yves Desfossés, « Apport des plans directeurs et de l'outil LiDAR aéroporté pour la caractérisation des impacts morphologiques de la Grande Guerre : exemple de la cote 108 (Berryau-Bac, France) », Géomorphologie : relief, processus, environnement, 2017 [En ligne], mis en ligne le 16 juin 2017, consulté le 10 juillet 2017. URL : http: [//geomorphologie.revues.org/11711]; Rémi DE Matos-Machado, Stéphanie Jacquemot, Gilles Arnaud-Fassetta, Francois Betard, Jean-Paul Амат, "Archéo-géomorphologie du champ de bataille de Verdun : les vestiges de la Grande Guerre sous l'œil du laser », actes du colloque du GMPCA, 2015, Besançon, à paraître. 